University of Nebraska - Lincoln

DigitalCommons@University of Nebraska - Lincoln

\title{
Numerical simulation of expansion and charring of carbon-epoxy laminates in fire environments
}

\author{
Matthew McGurn \\ University at Buffalo, the State University of New York (SUNY Buffalo), Buffalo, NY \\ Paul DesJardin \\ University at Buffalo, the State University of New York (SUNY Buffalo), Buffalo, NY \\ Amanda Dodd \\ Sandia National Laboratories, Albuquerque, NM
}

Follow this and additional works at: https://digitalcommons.unl.edu/usdoepub

Part of the Bioresource and Agricultural Engineering Commons

McGurn, Matthew; DesJardin, Paul; and Dodd, Amanda, "Numerical simulation of expansion and charring of carbon-epoxy laminates in fire environments" (2012). US Department of Energy Publications. 124. https://digitalcommons.unl.edu/usdoepub/124

This Article is brought to you for free and open access by the U.S. Department of Energy at DigitalCommons@University of Nebraska - Lincoln. It has been accepted for inclusion in US Department of Energy Publications by an authorized administrator of DigitalCommons@University of Nebraska - Lincoln. 


\title{
Numerical simulation of expansion and charring of carbon-epoxy laminates in fire environments
}

\author{
Matthew T. McGurn a , Paul E. DesJardin ${ }^{\mathrm{a}, *}$, Amanda B. Dodd ${ }^{\mathrm{b}}$ \\ a Department of Mechanical and Aerospace Engineering, University at Buffalo, the State University of New York (SUNY Buffalo), Buffalo, NY 14260-4400, United States \\ ${ }^{\mathrm{b}}$ Sandia National Laboratories, Albuquerque, NM 87185, United States
}

\section{A R T I C L E I N F O}

\section{Article history:}

Received 5 June 2011

Received in revised form 9 June 2011

Available online 3 October 2011

\section{Keywords:}

Modeling

Heat release rate

Carbon epoxy laminate

Composite swelling

\begin{abstract}
A B S T R A C T
A thermal model is developed for the response of carbon-epoxy composite laminates in fire environments. The model is based on a porous media description that includes the effects of gas transport within the laminate along with swelling. Model comparisons are conducted against the data from Quintiere et al. [34]. Verifications are conducted for both coupon level and intermediate scale one-sided heating tests. Comparisons of the heat release rate (HRR) and time-to-ignition as well as the final products (mass fractions, volume percentages, porosity, etc.) are conducted. Overall, the agreement between available the data and model is good considering the simplified approximations to account for flame heat flux. A sensitivity study using a newly developed swelling model shows the importance of accounting for laminate expansion for the prediction of burnout. Reasonable agreement is observed between the model and data of the final product composition that includes porosity, mass fractions and volume expansion ratio.
\end{abstract}

(c) 2011 Published by Elsevier Ltd.

\section{Introduction}

Composite materials are being used at an increasing rate in applications including aerospace vessels and other transport vehicles. The advantages of these structures are their high strength to weight ratio, corrosion resistance, and ease of fabrication. One of the safety challenges to application of composite materials is their susceptibility to fire. When exposed to fire, composites degrade, releasing volatile gases, and producing char, thereby reducing structural integrity. The reliance on composite materials for primary structural components only serves to increase the need for improved modeling techniques [1-7].

The modeling of composite materials in fire environments requires knowledge of the temperature field. One of the earliest models for the thermal response of composites is the work of Henderson et al. [8-10], where the composite material was modeled as composed of either virgin or char material. This modeling approach has been expanded and modified most notably by Sullivan and Salamon [11-13], Springer and colleagues [14-16], Dimitrienko [17-20], Gibson et al. [21], Mouritz et al. [22-24] and DiBlasi et al. $[25,26]$. Some of these models have been incorporated into several publicly available codes that include Gpyro by Lautenberger $[27,28]$, the solid phase model in the NIST Fire Dynamics

\footnotetext{
* Corresponding author. Tel.: +1 716645 1467; fax: +1 7166452883 .

E-mail address: ped3@buffalo.edu (P.E. DesJardin).
}

Simulator by McGrattan et al. [29], and ThermaKin by Stoliarov and Lyon [30,31]. For the present study, the thermo-mechanical pyrolysis model of Luo and DesJardin serves as the framework for modeling pyrolysis of a carbon-epoxy laminate [32]. Common between all these methods in modeling the temperature field is the inclusion of energy transport from heat conduction, polymer decomposition and volatile gas flow, however, the effects of volumetric swelling associated with pyrolysis is less common.

The effects of volumetric swelling on thermal response has been examined by Dimitrienko $[17,18]$, Springer et al. $[15,16]$ and Luo and DesJardin [32]. In these approaches swelling is modeled in the context of a micro-mechanics model using an effective thermal expansion coefficient that is deduced from experimental measurements. This approach is reasonable when the temperatures are below that associated with pyrolysis where elastic theories can be applied. At higher temperatures, however, the physical mechanisms of laminate swelling from char growth is quite complicated - involving fracture of the lamella, crack growth and propagation from gas expansion, and fiber fraying. In this study, a simpler phenomenological description is explored in which expansion is linearly correlated with a pyrolysis reaction progress variable - similar to the model of Staggs [33].

The thermal model is based on the extensive property data given by the study of Quintiere, Walters and Crowley (QWC) [34]. Quintiere and colleagues developed a complete set of properties carbon-fiber composite including but not limited to thermal properties, kinetics of degradation, and heat of decomposition. 
The data from QWC is used to construct phenomenological models of: (1) pyrolysis decomposition, (2) composite swelling and (3) thermal transport properties. Validation of these models are compared to the well documented coupon level and one-sided heating experimental results from QWC.

The remainder of the paper is as follows, an overview of the thermal modeling is provided in Section 2 with details of the composite swelling in Section 2.3. The finite element method utilized for the solution of the carbon-epoxy system is outlined in Section 2.5. Within Section 3, the computations of the response of coupon level samples are first conducted and compared to the mass loss, volumetric expansion, and time-to-ignition data of QWC. Further results include predictions of one-sided heating tests and are compared the experimental data of QWC. Comparisons are conducted of both the heat release rate (HRR) as well as the final products (mass fractions, volume percentages, porosity, etc.). Lastly conclusions from this study are summarized.

\section{Thermal modeling}

The material modeling for this effort is based on homogenization theories developed for both the thermal and mechanical fields of composite systems by Luo and DesJardin [32]. In this approach, the local governing equations within each constituent (e.g., fiber, resin, gas, char, etc.) are first defined. These equations, representing the local mass, thermal and mechanical response of that material, are assumed to be locally valid within a given constituent. These equations are then averaged over a localized volume. The volume is chosen to be sufficiently large relative to the mesoscopic features of the laminate, (e.g., a unit cell associated with the weave), but small relative to the system, i.e., the entire laminate structure. After averaging the transport equations, additional surface integral terms appear in the equations representing the interphase processes. These terms originate from commuting the averaging operator with differentiation and, in general, are unknown and problem specific.

The gas density and individual gas species are tracked through the phase averaged species mass conservation equations. The total number of gaseous species depends upon the complexity of the pyrolysis model. Phase-averaged species conservation equations are solved for the bulk density, $\rho_{g}$, and the mass fraction of the $k$ th gas constituent, $Y_{k, g}$,

$\frac{\partial\left(\phi_{g} \rho_{g}\right)}{\partial t}=\frac{\partial}{\partial x}\left(\rho_{g} \frac{K}{\mu_{g}} \frac{\partial p_{g}}{\partial x}\right)+\dot{m}_{i n t}^{\prime \prime \prime}$

$\frac{\partial\left(\phi_{g} \rho_{g} Y_{k, g}\right)}{\partial t}=\frac{\partial}{\partial x}\left(\rho_{g} Y_{k, g} \frac{K}{\mu_{g}} \frac{\partial p_{g}}{\partial x}\right)+\frac{\partial}{\partial x}\left[\rho_{g} \phi_{g} D_{k, m} \frac{\partial Y_{k, g}}{\partial x}\right]+\dot{m}_{k, v o l}^{\prime \prime \prime} \phi_{g}+\dot{m}_{k, i n t}^{\prime \prime \prime}$

where a Darcy's Law is employed to approximate the bulk gas transport, i.e., $\phi_{g} u_{g}=-\left(K / \mu_{g}\right) \partial p_{g} / \partial x$ requiring the specification of the permeability, K. Fick's Law of diffusion accounts for the effects of differential diffusion, i.e., $u_{k, \text { diff }}=-D_{k, m}\left(\partial Y_{k, g} / \partial x\right) / Y_{k, g}$ where $D_{k, m}$ is the effective binary diffusion coefficient for the $k^{\text {th }}$ species in the mixture. The term, $\dot{m}_{k, v o l}^{\prime \prime \prime}$, on the RHS accounts for the production or consumption of species from volumetric reactions within the gas phase (e.g., oxidation of pyrolysis gases within the material). The last terms, $\dot{m}_{i n t}^{\prime \prime \prime}=\sum \dot{m}_{k, i n t}^{\prime \prime \prime}$, account for the production of pyrolysis gases from endothermic decomposition and evaporation processes which occur at phase interfaces. For the solid phases, the species conservation equation simplifies once the density of a given phase is assumed to be constant, i.e., $\rho_{k, s} \partial \phi_{k, s} / \partial t=\dot{m}_{k, i n t}^{\prime \prime \prime}$. Thermal equilibrium among the solid, liquid and gas phases is assumed resulting in a single transport equation to describe energy transport,

$$
\begin{aligned}
\rho C_{P} \frac{\partial T}{\partial t}= & \frac{\partial T}{\partial x}\left[\rho_{g} C_{P, g} \frac{K}{\mu_{g}} \frac{\partial p_{g}}{\partial x}\right]+\frac{\partial}{\partial x}\left(k \frac{\partial T}{\partial x}\right) \\
& +\sum_{k=1}^{N_{g}}\left(\frac{\partial T}{\partial x} C_{P, k} \rho_{g} \phi_{g} D_{k, m} \frac{\partial Y_{k, g}}{\partial x}\right)+\frac{\partial\left(\phi_{g} p_{g}\right)}{\partial t} \\
& -\frac{\partial}{\partial x}\left(p_{g} \frac{K}{\mu_{g}} \frac{\partial p_{g}}{\partial x}\right)-\sum_{k=1}^{N} h_{k}\left(\phi_{g} \dot{m}_{k, v o l}^{\prime \prime \prime}+\dot{m}_{k, \text { int }}^{\prime \prime \prime}\right)
\end{aligned}
$$

where $\rho\left(=\sum \rho_{k} \phi_{k}\right)$ and $C_{P}\left(=\sum \phi_{k} \rho_{k} / \rho C_{P, k}\right)$ are the bulk density and specific heat, respectively. The terms on the RHS of Eq. (2) represent bulk advection (via the Darcy Law approximation), conduction, differential diffusion, pressure work, and oxidation/decomposition rate processes.

\subsection{Pyrolysis rate modeling}

For the purposes of constructing phenomenological Arrhenius based decomposition rates, a reaction progress variable, $\alpha$, is often introduced defined in terms of the solid mass, $m\left(=m_{r}+m_{f}+m_{c}\right)$, as,

$\alpha=\frac{m-m_{o}}{m_{e}-m_{o}}$

The subscript $o$ and $e$ represent the initial and final states of the solid, respectively. Assuming the fiber does not participate in the pyrolysis then the rates of decomposition of the resin (r), char (c) and gas (g) can be expressed directly in terms of changes in $\alpha$,

$$
\begin{aligned}
& \dot{m}_{r, \text { int }}^{\prime \prime \prime}=-\frac{1}{1-\tau}\left[\rho_{o}-\left(V_{e} / V_{o}\right) \rho_{e}\right] \dot{\alpha} \\
& \dot{m}_{c, \text { int }}^{\prime \prime \prime}=\frac{\tau}{1-\tau}\left[\rho_{o}-\left(V_{e} / V_{o}\right) \rho_{e}\right] \dot{\alpha} \\
& \dot{m}_{g, \text { int }}^{\prime \prime \prime}=\left[\rho_{o}-\left(V_{e} / V_{o}\right) \rho_{e}\right] \dot{\alpha}
\end{aligned}
$$

where $\dot{\alpha}$ is modeled using an Arrhenius rate law suggested by QWC as: $\dot{\alpha}=\frac{1-\alpha}{1-\mu} k(T)$ with $\mu=\left(m_{f}+m_{c}\right)_{e} / m_{o}$ being defined as the char fraction and $k$ given as $k=a_{p} \exp \left(-E_{a} / R T\right)$. Values for the activation energy $\left(E_{a}\right)$ and pre-exponential constant $\left(a_{p}\right)$ were determined by QWC using TGA data resulting in kinetic parameters of $E_{a}=182 \mathrm{~kJ} / \mathrm{mol}$ and $a_{p}=9.67 \times 10^{10} \mathrm{~s}^{-1}$. The quantity $V_{e} / V_{o}$ in Eq. (4) is the overall volumetric expansion ratio assuming complete charring and is set equal to a value of 2.2 to match the measurements of QWC. The quantity $\tau$ is the mass of char per unit mass of resin and is defined as:

$\tau \equiv \frac{\dot{m}_{c, \text { int }}^{\prime \prime \prime}}{\dot{m}_{r, \text { int }}^{\prime \prime \prime}}=\frac{V_{e}}{V_{o}} \frac{\left(\phi_{c} \rho_{c}\right)_{e}}{\left(\phi_{r} \rho_{r}\right)_{o}}$

which can be directly related to the residue fraction as,

$\tau=\frac{\rho_{o} \mu-\left[\rho_{f} \phi_{f}\right]_{o}}{\left[\phi_{r} \rho_{r}\right]_{o}}$

where $\rho_{o}, \rho_{f}$ and $\phi_{f, o}$ are given by QWC as $1530 \mathrm{~kg} / \mathrm{m}^{3}, 1230 \mathrm{~kg} / \mathrm{m}^{3}$ and 0.6 , respectively. Neglecting the mass of the initial gas then the initial fiber volume fraction is $\phi_{r, o}=0.398$ and gas volume fraction is $\phi_{g, o}=1-\phi_{r, o}-\phi_{f, o}=0.007$. Substituting the initial volume fractions and densities into Eq. (5) results in $\tau=0.17$ which is consistent with the reported value given by QWC of $0.20 \pm 0.05$. It is important to note that for modeling purposes, the values of $\mu$ and $\tau$ should be self-consistent. The heat of decomposition, required to calculate the energy consumed through pyrolysis, is given by QWC as $2.5 \times 10^{6} \mathrm{~J} / \mathrm{kg}$ of the original material.

\subsection{Matrix thermal properties}

One of the major modeling challenges is accurate models of the transport coefficients $K$ and $k$. In the current study, the 
conductivity of the composite is assumed isotropic and modeled using a curve fit to the data of QWC given as: $k=0.023 T^{0.46} I W /$ $\mathrm{m}^{2}-{ }^{\circ} \mathrm{C}$, where $T$ is measured in ${ }^{\circ} \mathrm{C}$ and $\mathrm{I}$ is the identify matrix.

The gas is modeled as flow through a bank of circular cylinders with diameter, $d_{f}$ for which the permeability is be estimated using the following correlation [35],

$K=\frac{\phi_{g}^{3} d_{f}^{2}}{C\left(1-\phi_{g}\right)^{2}}$

where $C=144$ if the tubes are cylindrical in shape. $d_{f}$ is set equal to $0.1 \mathrm{~mm}$ based on an estimate of the fiber toe diameter determined by dividing the thickness of the laminate $(3.2 \mathrm{~mm}$ ) by twice the total number of plies (16). The rational being that each layer is composed of overlapping toes in the weave and the resin rich region between layers is small relative to the weave thickness. The resulting initial and final permeability using this approach are $2.42 \times 10^{-17} \mathrm{~m}^{2}$ and $2.83 \times 10^{-10} \mathrm{~m}^{2}$, respectively, however, the upper bound of $K$ is clipped to a value of $1 \times 10^{-13}$ to avoid unnecessarily small time steps to maintain numerical stability. For the heat flux ranges used in this study, the results are insensitive to this factor as long as it is chosen to be greater then $1 \times 10^{-14}$.

The bulk specific heat of the matrix is modeled using a reaction progress variable description, $C_{P}(T, \alpha)=C_{P, o}(T)+\alpha\left(C_{P, e}(T)-C_{P, o}(T)\right)$, using temperature dependent virgin $\left(C_{P, o}(T)\right)$ and charred properties $\left(C_{P, e}(T)\right)$ from QWC given as,

$C_{P, 0}=0.75+0.0041 T \mathrm{~J} / \mathrm{kg}-K$

$C_{P, e}=0.84+0.0035 T \mathrm{~J} / \mathrm{kg}-K$

where $T$ is in ${ }^{\circ} \mathrm{C}$.

\subsection{Composite swelling}

As discussed by QWC, an overall volumetric expansion ratio of $V_{e} / V_{o}=2.2$ is observed in the experiments. As will be shown in the results, the effects of volumetric expansion has a pronounced effect on burn-out times because of the changes in gas volume fraction and the overall growth of the composite thickness. To account for these effects, an evolution equation for the $k$ th solid volume fraction can, in principle, be derived,

$\frac{D \phi_{k}}{D t}=\frac{\dot{m}_{k, \text { int }}^{\prime \prime \prime}}{\rho_{k}}+\dot{V}_{k, \text { exp }}$

where the subscript $k(=f, r, c)$ represents either fiber, resin or char and the corresponding source/sink term, $\dot{m}_{k, \text { int }}^{\prime \prime \prime}$, is given in Eq. (4). The second term on the RHS of Eq. (9) accounts for the increasing solid volume fraction from swelling (expansion) processes and can be directly related to the divergence of the $k^{\text {th }}$ solid material velocity, i.e., $\dot{V}_{k, \exp }=-\phi_{k} \frac{\partial u_{k}}{\partial x}$. To rigorously account for the effects of swelling requires a detailed analysis of the mechanical response of the structure to determine $u_{k}$ that, in turn, will depend on the thermal field (via, thermal expansion, fiber fraying, etc.). To model this coupled thermo-mechanical system, a presumed micomechanics description is often defined in the context of unit cell homogenization approaches [32]. The exact nature of the swelling process is, however, quite complicated and potentially difficult to validate experimentally therefore a simpler phenomenological approach is pursued. Similar to the work of Staggs [33], in which the density is calculated as linear interpolation of a progress variable, the solid volume fraction is directly expressed as a linear function of the reaction progress variable,

$\phi_{k}=\phi_{k, o}+\left(\phi_{k, f}-\phi_{k, o}\right) \alpha$

where the initial $\left(\phi_{k, o}\right)$ and final $\left(\phi_{k, f}\right)$ volume fractions of each solid constituent that are summarized in Table 1 below.
Table 1

Summary of the initial and final volume fractions of solid constituents.

\begin{tabular}{lll}
\hline$k$ & $\phi_{k, o}$ & $\phi_{k f}$ \\
\hline$f$ & 0.600 & 0.273 \\
$r$ & 0.393 & 0.000 \\
$g$ & 0.007 & 0.706 \\
$c$ & 0.000 & 0.021 \\
\hline
\end{tabular}

This simple model accounts for the leading order effects from composite swelling which is to decrease the solid volume fractions and increase the gas volume fraction that will be shown to be important in the results. In addition, the composite geometry change from swelling is also accounted for using a newly developed element expansion algorithm. In this approach, each element node located at position $x_{o}$ is first assigned a neighboring reference node, $x_{o, r e f}$. The distance between these nodes at the start of the simulation is defined as: $d_{o}=x_{o}-x_{o, r e f}$, as shown in Fig. 1 . As the expansion process proceeds the distance between the two nodes
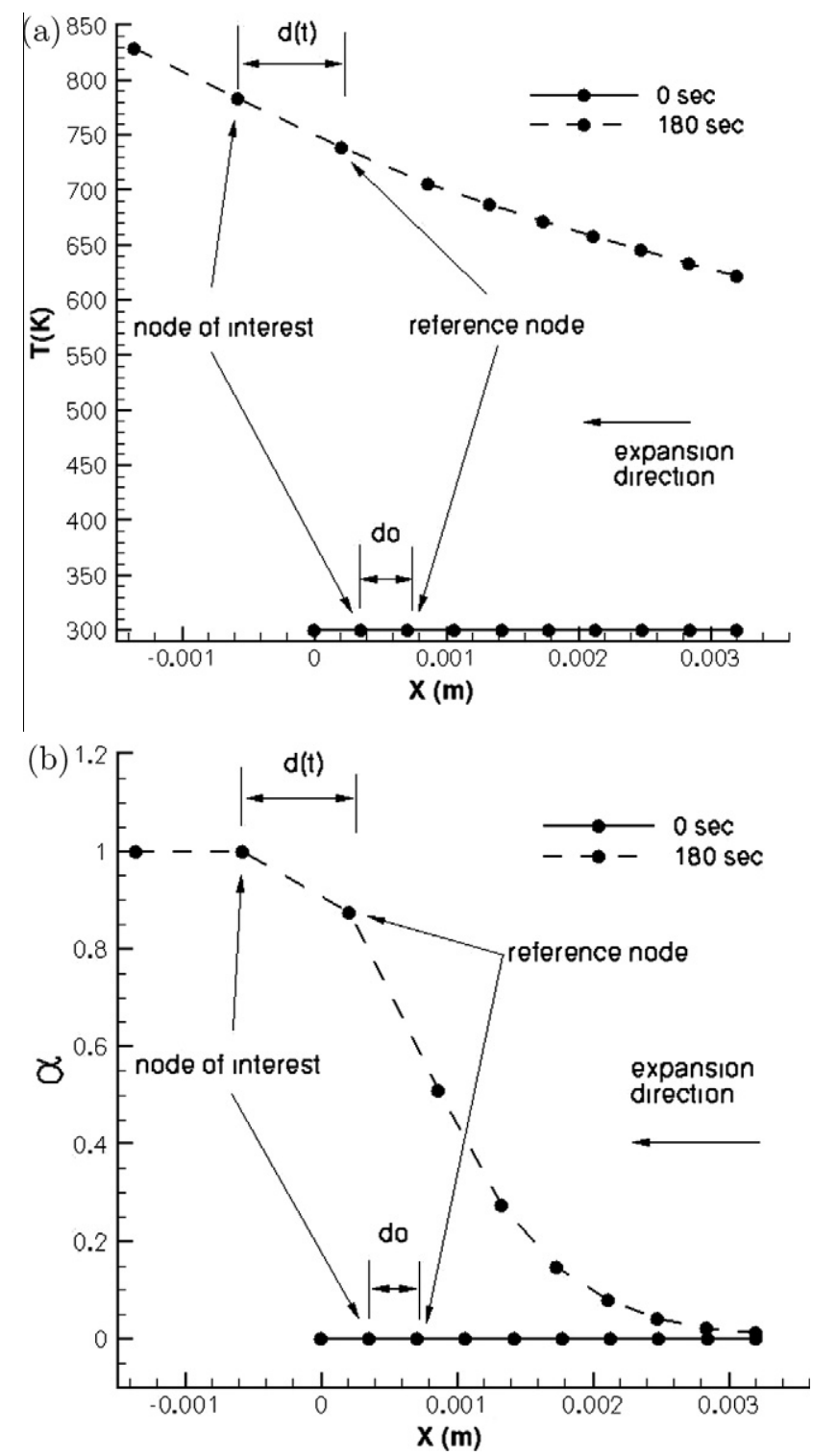

Fig. 1. Illustration of element expansion process in 1D for (a) temperature and (b) reaction progress variable at $t=0 \mathrm{~s}$ and $180 \mathrm{~s}$. 
is directly related to the expected volumetric expansion, $V / V_{o}$, via, $d=d_{o} V / V_{o}$, where $V / V_{o}$ can be determined by re-arranging Eq. (3),

$\frac{V}{V_{o}}=\frac{\left(\rho_{e} V_{e} / V_{o}-\rho_{o}\right) \alpha+\rho_{o}}{\rho}$

resulting in a non-linear dependence of $V / V_{o}$ on $\alpha$. In Eq. (11), $\rho=\sum \phi_{k} \rho_{k}$ is the total matrix density which is computed at each node. The neighboring reference node associated with each moving node is selected by a closest search in a direction that is opposite to that of a user prescribed expansion direction, $n_{\exp }=-d_{o} /\left|d_{o}\right|$. The user specified direction could, in general, be selected to point anywhere but for this study is chosen to point towards the boundary that is heated (i.e., the left boundary in Fig. 1). Element expansion is implemented after each integration time step by moving the element nodes as: $x(t)=x_{\text {ref }}(t)+d(t)$. The reference node shown in Fig. 1, is in turn, a function of its own reference node, and so on for the rest of the nodes. At the boundary furtherest from the heating source, the reference node and node of interest are the same, therefore $d_{o}=0$ and so the nodes farthest from the heated boundary do not move (i.e., the right boundary in Fig. 1). Ideally, the nodes furtherest away from the heating source should be updated first and the nodes closest to the heating source updated last. However, because of the unstructured nature of the data in the FE implementation, it is not straight forward to implement such an update strategy. Alternatively, a time linearization procedure is implemented such that, $x(t+\Delta t)=x_{\text {ref }}(t)+d(t+\Delta t)$, where $d(t+\Delta t)$ is determined as $d(t+\Delta t)=d_{o} V(t) / V_{o}$ with $V / V_{o}$ evaluated using Eq. (11). The resulting algorithm yields a smooth movement of nodes so that $V /$ $V_{o} \rightarrow V_{e} / V_{o}$ as $\alpha \rightarrow 1$.

Fig. 1 illustrates the element expansion algorithm for one-sided heating of a $3.2 \mathrm{~mm}$ thick carbon-epoxy laminate. The computations are one-dimensional using 10 elements across the through thickness. The right boundary is assumed adiabatic. A constant $60 \mathrm{~kW} / \mathrm{m}^{2}$ heat flux is imposed on the left boundary. The initial (a) temperature and (b) $\alpha$ are shown and after 3 min of heating. As expected, the temperature on the left face of the laminates increases for which decomposition of the laminate occurs as shown by the increase in $\alpha$. The expansion direction $\left(n_{\text {exp }}\right)$ denoted in the figures is directed to the left as that is direction of the applied heat. For illustration purposes, the second node in from the left boundary is identified as the node of interest and its self-identified reference node is the neighboring node to the right. As shown, the distance between them $(d)$ grows with time in accordance with Eq. (11) such that $|d| /\left|d_{o}\right|$ and approaches a final value of 2.2 corresponding to $V_{e} / V_{o}$.

\subsection{Gas properties and exothermic chemical reactions}

The mixture of pyrolysis gases and air are treated as ideal gases using CHEMKIN polynomial fits for thermodynamic properties [36]. The decomposition the epoxy resign is a complicated process in which a large number of species are released. The hydrocarbon chains in epoxy can either be broken through chain scission or side chain/group elimination [37]. However in the decomposition process new chemical bounds may be formed through additional cross-linking and cyclization [38]. In epoxy this process can result in the release of phenol, 4-isopropylphenol, bisphenol A, 4-t-butylo-cresol, and additional products not yet identified [37]. As an approximation proportions of carbon, hydrogen and oxygen in the pyrolysis gas are assumed to be the same as those given by Tewarson as $\mathrm{CH}_{1.3} \mathrm{O}_{0.2}$ [39]. For lack of data, the sensible enthalpy and specific heats of the pyrolysis product gas are assumed to be that of methane $\left(\mathrm{CH}_{4}\right)$ since the molecular weights are similar (i.e., 16.5 vs. 16). Once the pyrolysis gas leaves the composite, it is assumed to burn according to the following one-step molar reaction.

$$
\begin{aligned}
& \mathrm{CH}_{1.3} \mathrm{O}_{0.2}+1.225\left(\mathrm{O}_{2}+3.76 \mathrm{~N}_{2}\right) \\
& \quad \rightarrow \mathrm{CO}_{2}+0.65 \mathrm{H}_{2} \mathrm{O}+1.225(3.76) \mathrm{N}_{2}
\end{aligned}
$$

Using a suggested heat of combustion of $\Delta h_{C}=28.8 \mathrm{~kJ} / \mathrm{g}$ by Tewarson [39], a calculated heat of formation for the pyrolysis gas using Eq. (12) is $h_{\mathrm{CH}_{13} \mathrm{O}_{02}}^{\circ}=-4.5785 \mathrm{~kJ} / \mathrm{g}$ resulting in an adiabatic flame temperature of $T_{a d}=2300 \mathrm{~K}$.

\subsection{Numerical formulation}

The Finite Element (FE) numerical method is utilized to solve the density, species mass fraction and energy equations presented in Eqs. (1a), (1b), and (2), respectively. The solution procedure utilized standard Galerkin formulation consisting of linear basis functions.

\subsubsection{Weak formulation}

The application of the finite element solution procedure requires a weak form of the governing equations that is obtained by multiplying by a weight function $w$ and integrating over the domain $(\Omega)$ resulting in

$$
\begin{gathered}
\int_{\Omega} w \phi_{g} \frac{\partial \rho_{g}}{\partial t} d \Omega+\int_{\Omega} \frac{p_{g}}{\mu_{g}} \frac{\partial w}{\partial x}\left(K \frac{\partial \rho_{g}}{\partial x}\right) d \Omega=-\int_{\Omega} \frac{\rho_{g}^{2}}{\mu_{g}} \frac{\partial w}{\partial x}\left(K \frac{\partial R_{g} T}{\partial x}\right) d \Omega \\
\quad-\int_{\Gamma} w \dot{m}_{g}^{\prime \prime} n d \Gamma+\int_{\Omega} w \dot{m}_{i n t}^{\prime \prime \prime} d \Omega-\int_{\Omega} w \rho_{g} \partial \phi_{g} / \partial t d \Omega \\
\int_{\Omega} w \phi_{g} \rho_{g} \frac{\partial Y_{k, g}}{\partial t} d \Omega+\int_{\Omega} w \rho_{g} \phi_{g} u_{g} \frac{\partial Y_{k, g}}{\partial x} d \Omega+\int_{\Omega} \rho_{g} \phi_{g} D_{k, m} \frac{\partial Y_{k, g}}{\partial x} \frac{\partial w}{\partial x} d \Omega \\
=-\int_{\Gamma} w \dot{m}_{k, g}^{\prime \prime} n d \Gamma+\int_{\Omega} w\left(\dot{m}_{k, v o l}^{\prime \prime \prime} \phi_{g}+\dot{m}_{k, i n t}^{\prime \prime \prime}-Y_{k, g} \dot{m}_{i n t}^{\prime \prime \prime}\right) d \Omega \\
\int_{\Omega} w\left(\rho C_{P}-\phi_{g} \rho_{g} R_{g}\right) \frac{\partial T}{\partial t} d \Omega+\int_{\Omega} w\left(\rho_{g} \phi_{g} C_{P, g} u_{g}-u_{g} \phi_{g} \rho_{g} R_{g}\right) \frac{\partial T}{\partial x} d \Omega \\
+\int_{\Omega}\left(k \frac{\partial T}{\partial x}\right) \frac{\partial w}{\partial x} d \Omega \\
=-\int_{\Omega} w T\left[\frac{\partial\left(u_{g} \phi_{g} \rho_{g} R_{g}\right)}{\partial x}+\frac{\partial\left(\phi_{g} \rho_{g} R\right)}{\partial t}\right] d \Omega \\
-\int_{\Omega} w \sum_{k=1}^{N} h_{k}\left(\phi_{g} \dot{m}_{k, v o l}^{\prime \prime \prime}+\dot{m}_{k, i n t}^{\prime \prime \prime}\right) d \Omega \\
-\int_{\Gamma} w q n d \Gamma
\end{gathered}
$$

where $\Gamma$ and $n$ define the boundary and outward normal of the domain. Eq. (13c) assumes energy transport due to molecular diffusion is negligible which is a reasonable assumption since conduction through the matrix is the dominant method of heat transfer. The second $\left(\phi_{g} \rho_{g} R_{g}\right)$ and seventh $\left(\partial\left(\phi_{g} \rho_{g} R\right) / \partial t\right)$ terms in Eq. (13c) are a result of the substitution of the ideal gas equation of state into the temporal pressure derivative in Eq. (2). Lagrange interpolating polynomial are chosen for the spacial approximation of $\rho_{g}, Y_{k, g}$ and $T$ [40]. For a given element, defined by $N$ nodes, the field variable $\chi\left(=\rho_{g}, Y_{k, g}, T\right)$ is assumed to fit the following Lagrange interpolating polynomial,

$\chi(x, t) \approx \chi^{e}(x, t)=\sum_{j=1}^{N} \chi_{j}^{e}(t) \psi_{j}^{e}(x)$.

In order to close the system of equations produced with the substitution of Eq. (14) into the weak form of the governing equations, Eq. (13), $\mathrm{N}$ expressions for the weighting function must be chosen. The required weighting functions are chosen to be equal to the basis functions used to describe the field varaiable such that 
$w_{i}=\psi_{1}, \psi_{2}, \ldots \psi_{N}$. Substituting the approximations given in Eq. (14) and basis function for $w$ the weak form of the governing equations can be written in matrix form as,

$$
\begin{aligned}
& {[M]\left\{\dot{\rho}_{g}\right\}+[K]\left\{\rho_{g}\right\}=\{Q\}+\left\{q_{b}\right\}} \\
& M_{i j}=\int_{\Omega_{e}} \psi_{i}^{e} \psi_{j}^{e} \phi_{g} d \Omega \\
& K_{i j}=\int_{\Omega_{e}} \frac{p_{g}}{\mu_{g}} \frac{\partial \psi_{i}^{e}}{\partial x}\left(K \frac{\partial \psi_{j}^{e}}{\partial x}\right) d \Omega \\
& Q_{i}=\int_{\Omega_{e}} \psi_{i}^{e}\left(\dot{m}_{i n t}^{\prime \prime \prime}-\rho_{g} \partial \phi_{g} / \partial t\right) d \Omega-\int_{\Omega_{e}} \frac{\rho_{g}^{2}}{\mu_{g}} \frac{\partial \psi_{i}^{e}}{\partial x}\left(K \frac{\partial R_{g} T}{\partial x}\right) d \Omega \\
& q_{b, i}=-\int_{\Gamma_{e}} \psi_{i}^{e} \dot{m}_{g}^{\prime \prime} n d \Gamma \\
& {[M]\left\{\dot{Y}_{k, g}\right\}+[K]\left\{Y_{k, g}\right\}=\{Q\}+\left\{q_{b}\right\}} \\
& M_{i j}=\int_{\Omega_{e}} \psi_{i}^{e} \psi_{j}^{e} \phi_{g} \rho_{g} d \Omega \\
& K_{i j}=\int_{\Omega_{e}} \rho_{g} \phi_{g} D_{k, m} \frac{\partial \psi_{i}^{e}}{\partial x} \frac{\partial \psi_{j}^{e}}{\partial x} d \Omega+\int_{\Omega_{e}} \psi_{i}^{e} \rho_{g} \phi_{g} u_{g} \frac{\partial \psi_{j}^{e}}{\partial x} d \Omega \\
& Q_{i}=\int_{\Omega_{e}} \psi_{i}^{e}\left(\dot{m}_{k, v o l}^{\prime \prime \prime} \phi_{g}+\dot{m}_{k, i n t}^{\prime \prime \prime}-Y_{k, g} \dot{m}_{i n t}^{\prime \prime \prime}\right) d \Omega \\
& q_{b, i}=-\int_{\Gamma_{e}} \psi_{i}^{e} \dot{m}_{k, g}^{\prime \prime} n d \Gamma \\
& {[M]\{\dot{T}\}+[K]\{T\}=\{Q\}+\left\{q_{b}\right\}} \\
& M_{i j}=\int_{\Omega_{e}}\left(\rho C_{P}-\phi_{g} \rho_{g} R_{g}\right) \psi_{i}^{e} \psi_{j}^{e} d \Omega \\
& K_{i j}=\int_{\Omega_{e}} k\left(\frac{\partial \psi_{i}^{e}}{\partial x} \frac{\partial \psi_{j}^{e}}{\partial x}\right)+\psi_{i}^{e}\left(\rho_{g} \phi_{g} C_{P, g} u_{g}-u_{g} \phi_{g} \rho_{g} R_{g}\right) \frac{\partial \psi_{j}^{e}}{\partial x} \\
& -\left[\frac{\partial}{\partial x}\left(u_{g} \phi_{g} \rho_{g} R_{g}\right)+\frac{\partial\left(\phi_{g} \rho_{g} R_{g}\right)}{\partial t}\right] \psi_{i}^{e} \psi_{j}^{e} d \Omega \\
& Q_{i}=\int_{\Omega_{e}} \sum_{i=1}^{N} h_{i}\left(\phi_{g} \dot{m}_{k, v o l}^{\prime \prime \prime}+\dot{m}_{k, \text { int }}^{\prime \prime \prime}\right) \psi_{i}^{e} d \Omega \\
& q_{b, i}=-\int_{\Gamma_{e}} \psi_{i}^{e} q \operatorname{qnd} \Gamma
\end{aligned}
$$

\subsubsection{Numerical integration in time}

The integration of Eq. (15), is performed utilizing the alpha family integration scheme the details of which can be obtained in Ref. [40]. Eqs. (15a), (15b), and (15c) are solved separately. However, due to the nonlinear relationship between $\rho_{g}, Y_{k, g}$ and $T$ this assumption can lead to considerable errors and numerical instability for highly transient processes. This is remedied with the introduction of a Point Successive Over-Relaxation (PSOR) method where within a single time step the solution is sub cycled.

The explicit source terms $\dot{m}_{k, i n t}^{\prime \prime \prime}, \dot{m}_{k, v o l}^{\prime \prime \prime}$ and $\partial \phi_{g} / \partial t$ involving the conversion from one phase/species to another are solved using a fractional step method to mitigate the numerical stiffness associated with integrating Arrhenius based reaction rates. In this approach, the source terms in Eq. (15) are integrated over the time step, $\Delta t$, using a separate ODE solver assuming the rest of the transport processes are "frozen". Source terms are then constructed from the ODE result and substituted into the finite element solver.

\subsubsection{Numerical integration in space}

The calculation of the elemental matrix presented in Eq. (15) requires the integration over element domain $\Omega_{e}$. This procedure is accomplished by mapping to a master element and approximating the integration using Gauss quadrature integration [40], where the integration is approximated as a sum of the weights multiplied by the integrand evaluated at the quadrature locations. Standard implementation of this approximation, however results in nonphysical pressure oscillations in the solution. The root of the problem is the linearization of the pressure gradient term (as a function of $\rho_{g}$ and $T$ ) which may result in non-zero values for a uniform pressure. To rectify this problem, the coefficients $\frac{\rho_{g} R_{g} T K}{\mu_{g}}$ and $\frac{\rho_{g}^{2} K}{\mu_{g}}$ in Eq. (1a) are assumed uniform throughout the element and ${ }^{\mu_{g}}$ are determined by interpolating to the element center. This implementation satisfies the limitings case of uniform pressure, prescribed by a increasing temperature in space and corresponding decrease in density, as dictated by the ideal gas law.

\section{Results}

Three sets of data are used from QWC consisting of coupon scale TGA data, time-to-ignition, and one-sided heating, calorimeter tests. In the one-sided heating tests, $15 \times 15 \mathrm{~cm}$ samples are heated by a radiant heater. The experiments are carried out according to Title 14 Code of Federal Regulations (CFR) 25.853 a-1 which is the Federal Aviation Administration (FAA) flammability test method for large surface area materials for aircraft cabin interiors. Reported values of heat release rate (HRR), final volume expansion ratio $\left(V / V_{o}\right)$, thickness expansion ratio, final porosity $\left(V_{g} / V_{e}\right)$, char + resin mass to initial resin mass ratio $\left(Y_{r c}=\left(M_{c}+M_{r}\right)_{e} / M_{r, o}\right)$ and fraction of remaining mass $\left(Y_{r c f}\right)$ are given by QWC.

\subsection{Coupon scale validation}

The heating rates selected for the simulations are 1, 3, 10 and $30^{\circ} \mathrm{C} / \mathrm{min}$ to match those of QWC and compare the sample mass fraction to the TGA data. The bulk solid mass fraction, $Y_{r c f}=M_{s} /$ $M_{s, o}=\left(M_{r}+M_{c}+M_{f}\right) / M_{s, o}$, is computed by integrating the masses of each constituent element-wise $\left(\Omega^{e}\right)$ over the solution domain $(\Omega)$ using the following relation,

$$
\begin{aligned}
M_{k} & =\int_{\Omega} \phi_{k} \rho_{k} d V \simeq \sum_{e} \int_{\Omega_{e}} J(\zeta) \phi_{k}(\zeta) \rho_{k}(\zeta) d V^{*} \\
& =\sum_{e} \sum_{j} W_{j}\left(\zeta_{j}\right) J\left(\zeta_{j}\right) \phi_{k}\left(\zeta_{j}\right) \rho_{k}\left(\zeta_{j}\right)
\end{aligned}
$$

where $J$ is the time varying Jacobian associated with the mapping of the physical space $(x)$ to the reference computational space $(\zeta)$ as the elements stretch due to swelling expansion processes. The quantities $W_{j}$ and $\zeta_{j}$ are the quadrature weights and locations in mapped space on the element for the numerical integration.

One-dimensional simulations are conducted using prescribed time dependent temperature boundaries. The temperature of the boundaries are increased in time to be consistent with the prescribed heating rate. A total of 10 elements are used to discretize the $3.2 \mathrm{~mm}$ thickness sample which is deemed adequate based on a grid sensitivity check (not shown). Fig. 2(a) shows instantaneous snapshots of temperature (solid lines) and $\alpha$ (dashed lines) for the $10^{\circ} \mathrm{C} / \mathrm{min}$ heating case at $t=775,1775$ and $2025 \mathrm{~s}$. The temperature is nearly uniform indicating that a thermally lumped analysis for developing the kinetic rates is valid. Fig. 2(b) shows the solid mass fraction compared to TGA results for heating rates of $3{ }^{\circ} \mathrm{C} / \mathrm{min}, 10^{\circ} \mathrm{C} / \mathrm{min}$ and $30^{\circ} \mathrm{C} / \mathrm{min}$. As shown, the overall agreement is very good - demonstrating that the Arrhenius kinetics are properly incorporated into the framework.

\subsection{Time-to-ignition simulations}

To further validate the models transient behavior against data, one-dimensional simulations are conducted using 30 elements for grid independent results. The time-to-ignition is estimated based upon a critical mass flow calibrated from experimental data of QWC. It is assumed that this critical mass flow is sufficient is 

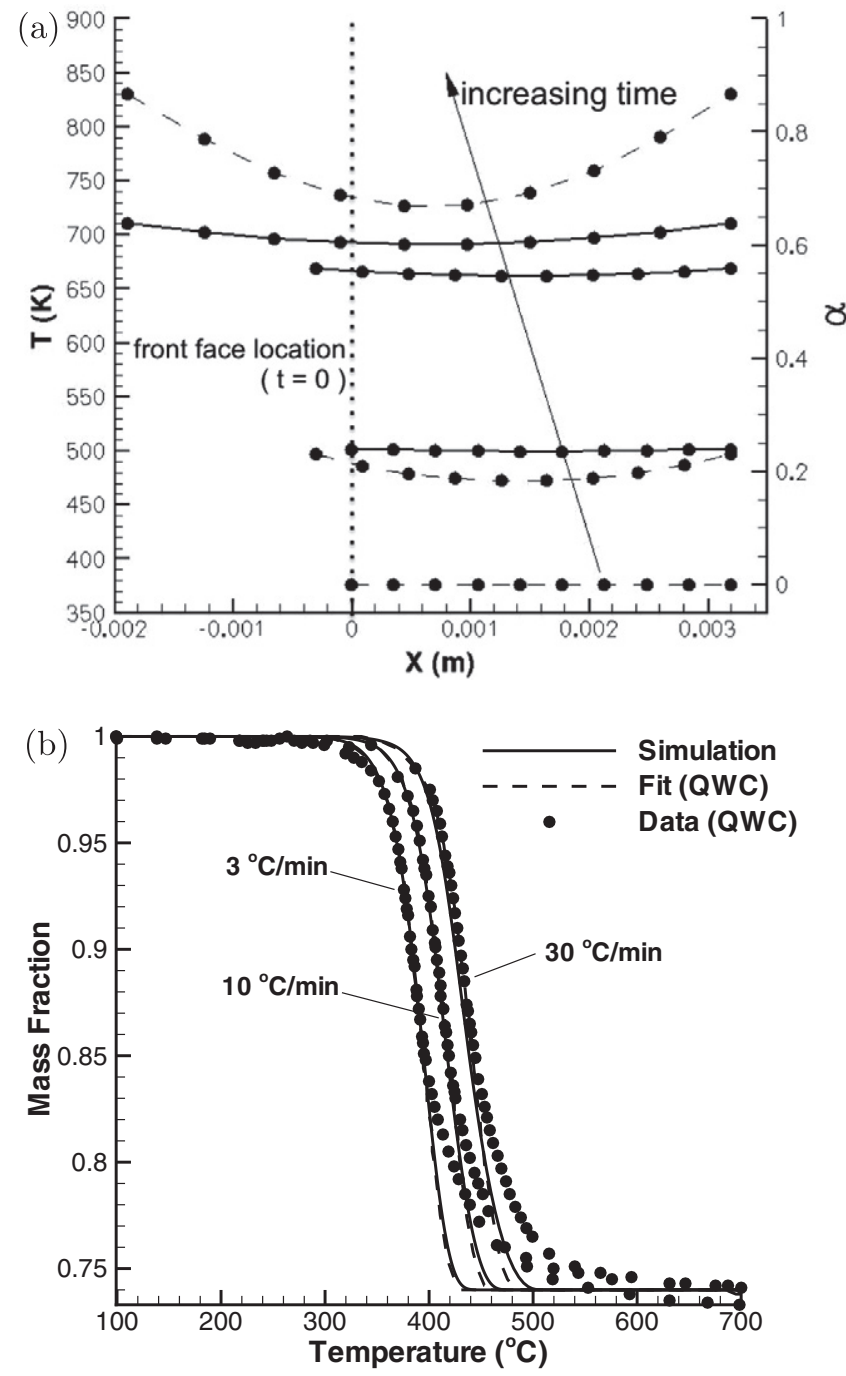

Fig. 2. Coupon scale simulations showing (a) instantaneous snapshots of temperature (solid lines) and $\alpha$ (dashed lines) at $t=775,1775$ and $2025 \mathrm{~s}$ for a $10^{\circ} \mathrm{C} / \mathrm{min}$ heating rate and (b) comparison of predictions of solid mass fraction (solid lines) to TGA data from Quintiere et al. [34] (symbols) and their curve fit using a first-order Arrhenius rate model.

providing fuel to produce a flame off the surface. The critical mass flux was calibrated from ignition times of the rough side of the composite (with pilot flame) provided by QWC, summarized in Fig. 3.

The thermal boundary conditions for the left (L) and right (R) boundaries are treated by defining the net heat flux in terms of incident (i), convective and surface blowing fluxes.

$\dot{q}_{L}^{\prime \prime}=\dot{q}_{i}^{\prime \prime}+h\left(T_{\infty, L}-T_{L}\right)-\left.\dot{m}_{g, n}^{\prime \prime} h_{g}\right|_{L}$

$\dot{q}_{R}^{\prime \prime}=h\left(T_{\infty, R}-T_{R}\right)-\left.\dot{m}_{g, n}^{\prime \prime} h_{g}\right|_{R}$

where $\dot{q}^{\prime \prime}=-q n$ and $\dot{m}_{g, n}^{\prime \prime}=\dot{m}_{g}^{\prime \prime} n$ with $n$ being the outward normal from the solid surface $(n=-1,1)$. The convective coefficients for the left and right boundaries are assumed to be constant and equal to $h=10 \mathrm{~W} / \mathrm{m}^{2} \mathrm{~K}$. The far-field temperatures on the surfaces are assumed constant and equal to $300 \mathrm{~K}$. For the gas transport, the gas density is determined on the left boundary assuming a constant pressure, via an equation of state and calculated surface temperature. For the mass fractions, $Y_{k}$, given in Eq. (1b), the following outflow convective boundary condition is imposed.

if $u_{g, n}>0$ then $D Y_{k} / D t=0$ else $Y_{k}(t+\Delta t)=Y_{k}(t)$, where $u_{g, n}=u_{g} n$. The specification of the boundary conditions for gas transport on the right boundary is less certain. While aluminum foil was used to seal the back surfaces in the experiments, it is not clear that it provided an effective seal since tests with and without the foil showed little difference in the resulting flame observed jetting out the front face [34]. To explore the sensitivity of the calculations to this uncertainty, cases are conducted using both open (i.e., constant pressure and the convective boundary of Eq. (17)) and closed (sealed) right boundaries. For the closed cases the flux for the gases on the right boundary are set equal to zero.

In lieu of a coupled fluid combustion model a critical mass flux criteria is utilized to estimate the time-to-ignition. This criterion requires that the critical mass flux be calibrated for each simulation, sealed and open right boundaries. The applied heat flux at the calibration point was chosen from the available experimental data as the minimum applied heat flux that could support piloted ignition. This point, shown in Fig. 3, corresponds to the time-toignition from QWC data for an applied heat flux of $19.5 \mathrm{~kW} / \mathrm{m}^{2}$. For this heat load the time-to-ignition is $330 \mathrm{~s}$. At $330 \mathrm{~s}$ the predicted mass fluxes are $3.35 \mathrm{~g} / \mathrm{m}^{2} \mathrm{~s}$ (sealed) and $2.40 \mathrm{~g} / \mathrm{m}^{2} \mathrm{~s}$ (open), respectively. The predicted critical mass fluxes are used to estimate time-to-ignition for the rest of the applied heat loads of $10,17.5$, $25,31.6,50,75$, and $100 \mathrm{~kW} / \mathrm{m}^{2}$.

Fig. 3 shows comparisons of the simulation results to measurements for time-to-ignition. The differences between the predictions and measurements are $7.4 \%, 5.9 \%, 21.4 \%$, and $38.2 \%$ for applied heat fluxes of $25,50,75$, and $100 \mathrm{~kW} / \mathrm{m}^{2}$, respectively, which is within experimental uncertainty for the lower heating rates. The time-to-ignition predictions using the open boundary condition are slightly lower then the sealed cases due to the gas mass loss through the right boundary.

\subsection{Intermediate scale validation using one-sided heating experiments}

One-sided heating simulations are conducted using a flame heat flux model and compared against the available data. Measured bulk quantities of the final products are directly computed in the FE model by integrating over the entire solution domain similar to that for the coupon level samples. To estimate the heat release rate, it is assumed that the mass flux blowing off of the heated surface instantly burns with the surrounding air. The heat release rate per unit surface area may then be estimated as: $H R R=\left.\dot{m}_{g, n}^{\prime \prime}\right|_{L} \Delta h_{C}$, where $\left.\dot{m}_{g, n}^{\prime \prime}\right|_{L}$ is the mass flux from the heated left surface of the FE model. In this approximation all mass that is released is assumed to contribute to the HRR after the critical mass flux is reached. The experimental measurements of HRR, however, consist of primarily the convective contributions of the HRR and therefore it is expected that the model will over-predict the peak HRR.

The thermal boundary conditions are defined as they are in Eqs. (16a) and (16b), however, the far-field temperature on the left surface is assumed equal to $300 \mathrm{~K}$ until ignition when $\left.\dot{m}_{g, n}^{\prime \prime}\right|_{L}>\dot{m}_{c r i t}^{\prime \prime}$. The value of $\dot{m}_{\text {crit }}^{\prime \prime}$ is either $3.35 \mathrm{~g} / \mathrm{m}^{2} \mathrm{~s}$ (sealed) or $2.40 \mathrm{~g} / \mathrm{m}^{2} \mathrm{~s}$ (open). After ignition the far-field temperature is set to $T_{\infty, L}=0.5\left(T_{a d}+T_{L}\right)$ to account for the additional heat flux from the near-wall flame with $T_{a d}=2300 \mathrm{~K}$ determined from Eq. (12). After sufficient burning, the $\left.\dot{m}_{g, n}^{\prime \prime}\right|_{L}$ again falls below $\dot{m}_{c r i t}^{\prime \prime}$ and the flame is assumed to extinguish. When this occurs the left far-field temperature is reset to $T_{\infty, L}=300 \mathrm{~K}$. The gas transport boundary conditions are treated identically to the time-to-ignition cases where both the open and sealed right boundaries are examined.

Fig. 4 shows comparisons of the heat release rate for four different heating rates of (a) $\dot{q}_{i}^{\prime \prime}=25$, (b) 50 , (c) 75 and (d) $100 \mathrm{~kW} / \mathrm{m}^{2}$. For all cases, the right boundary is assumed open. For each heat flux case, simulations are conducted both with (solid lines) and without (dashed lines) the expansion model activated and are compared to data (symbols) consisting of 4 to 5 separate runs. 


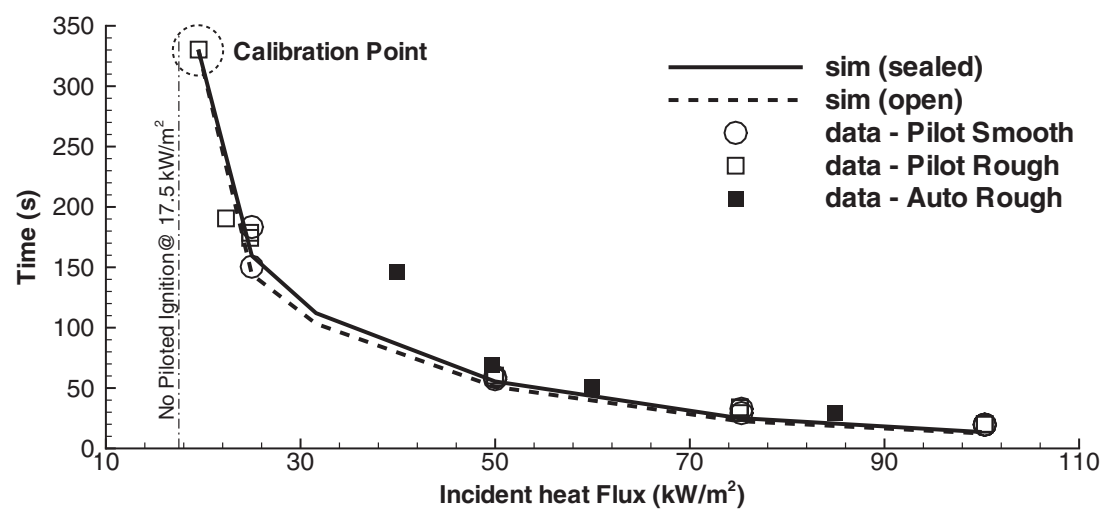

Fig. 3. Time-to-ignition cases with simulated sealed and open right boundaries compared against time-to-ignition data provided by QWC.

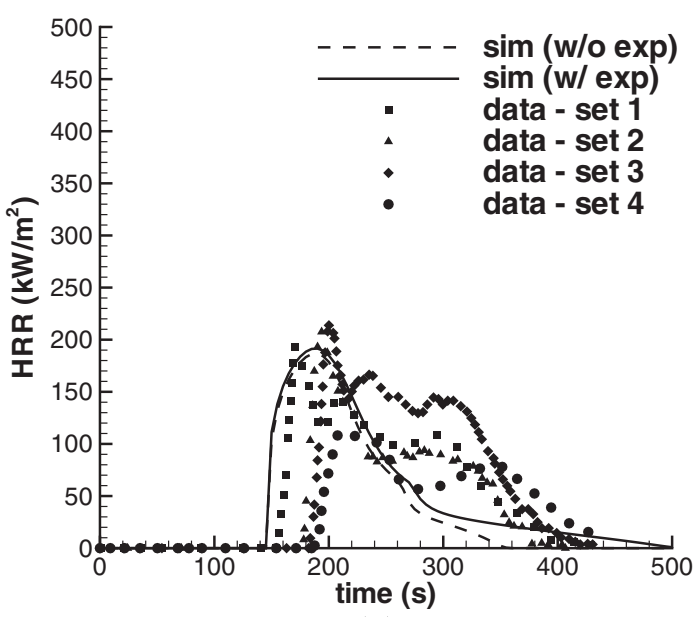

(a)

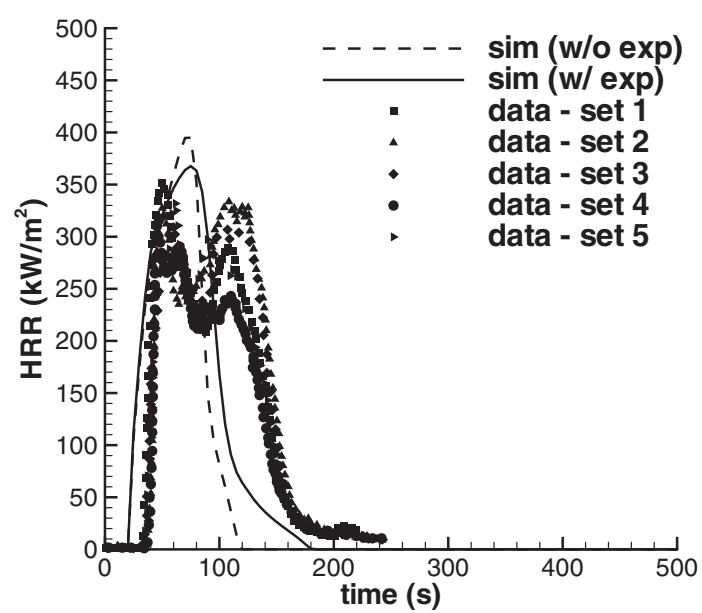

(c)

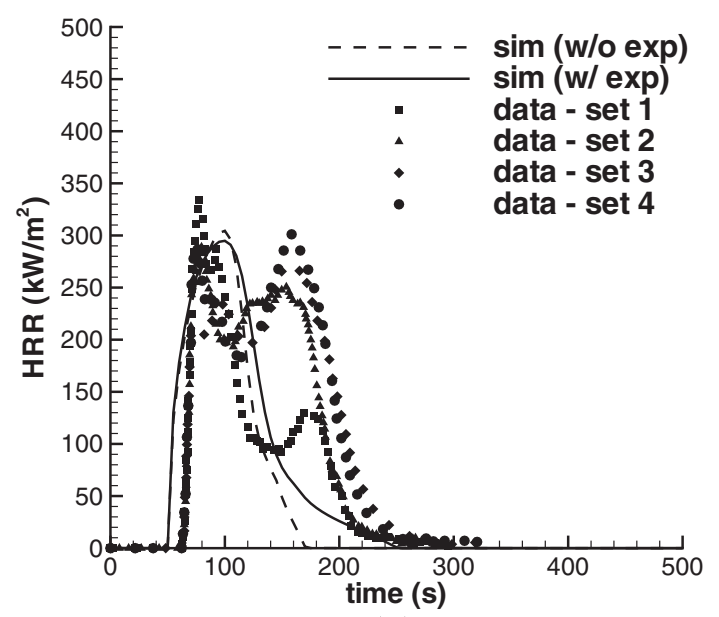

(b)

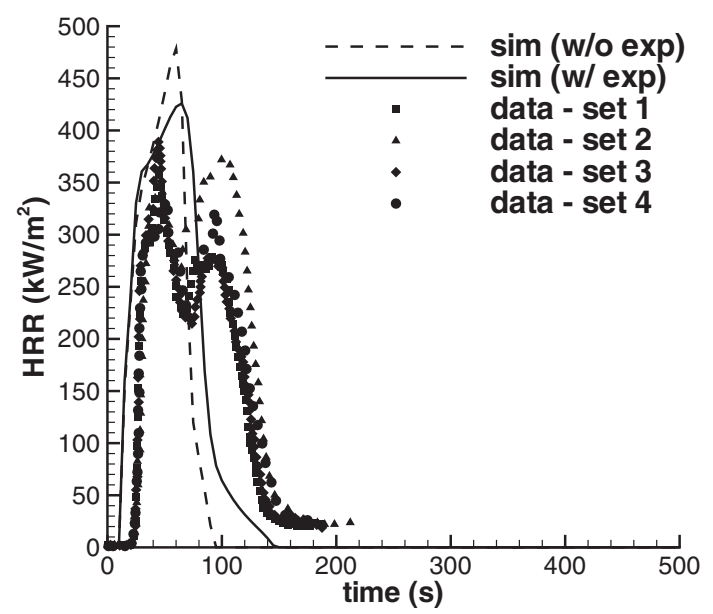

(d)

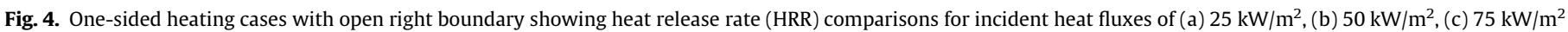
and (d) $100 \mathrm{~kW} / \mathrm{m}^{2}$.

Overall the agreement of the model to the data is reasonable considering the simplifications in estimating the heat release rate and the variability in the experimental data. In all cases, the model under-predicts ignition times with errors consistent with the ignition time predictions of Fig. 3. Comparing simulation cases without $(\mathrm{w} /$ o) and with $(\mathrm{w} /)$ the expansion model shows that accounting for volumetric expansion processes extends the burnout time by at least $50 \%$ for all cases resulting in much better agreement to the data. The reason for the premature burnout times w/o expansion is due to the effective reduced mass of the sample by using the final volume fractions summarized in Table 1 without increasing the overall size of the sample.

While the peak heat flux and burn out times predicted are reasonable in Fig. 4, the duration and overall heat release, $\Delta Q=$ 
$\int_{t} H R R(t) d t$, are significantly smaller than the experiments. This may be expected since the gas escaping out the right boundary was not accounted for in the overall heat release. To explore this possibility, additional simulations are conducted sealing the right boundary. Fig. 5 are the same cases as those of Fig. 4 but with the right boundary sealed. It is apparent that the sealing of the right boundary has a pronounced effect on $\Delta Q$ as well as the time history of HRR. A secondary plateau in heat release is observed that is more consistent with the double peak HRR history observed in the experiments. Since the simulations with the expansion model with open (Fig. 4) and sealed (Fig. 5) appear to bound the HRR data, it is reasonable to assume that the actual boundary from the experiments lies between these limits. This observation shows the importance of properly characterizing the unheated boundary for model validation purposes - the easiest approach may be to simply leave the unheated surface completely open or insulated with a very porous thermal blanket (e.g., superwool).

QWC offers an explanation for the twin peak HRR in Figs. 4 and 5. They attribute the appearance of the first peak to the composite seeking to achieve a steady-state thermal distribution for the resin binder as an insulation layer is built up and the second peak to thermal heating wave reaching the back of the composite and being inhibited thereafter from the insulation backer board. A more quantitative complementary explanation can be explored by examining the instantaneous distributions of through thickness temperature and pressure. Fig. 6 shows snapshots of (a) $T \& \alpha$ and (b) $p_{g} \& K$ at $t=25,50$ and $150 \mathrm{~s}$. The temperature smoothly rises with increasing time as the decomposition front penetrates deeper into the laminate (denoted by $\alpha$ ). The gas pressure, however, shows a very different behavior with a local peak pressure at the decomposition front where the permeability is low. The pressure continues to grow with time to a peak value of $1.5 \mathrm{~atm}$ until the decomposition front reaches the back face of the laminate at $t=75 \mathrm{~s}$. Between $75 \mathrm{~s}$ and $150 \mathrm{~s}$ the residual resin decomposes resulting in a capacitance of pressure that slowly vents off creating the plateau region of Fig. 5(d). While the current model qualitatively captures the double peak history, the predicted second peak in HRR is lower than measured in the experiments. The reasons for this are twofold. The first is the simplified estimate of HRR that does not account for the effects of turbulent mixing already discussed. Future fully coupled simulations will explore relaxing this assumption. The second reason is the limitations of the current permeability model. The exact time history of the venting process out the front face will be very sensitive to how the permeability is modeled. As shown in Fig. 6(b), the model for permeability results in a fairly monotonic permeability profile through the decomposition front (even though it is a strong non-linear function of porosity, see Eq. (7)). If, however, the permeability decreases abruptly in

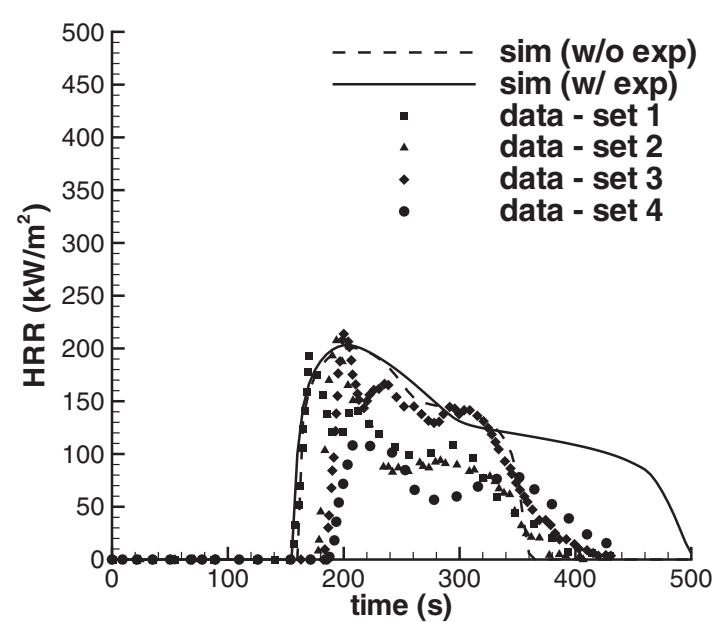

(a)

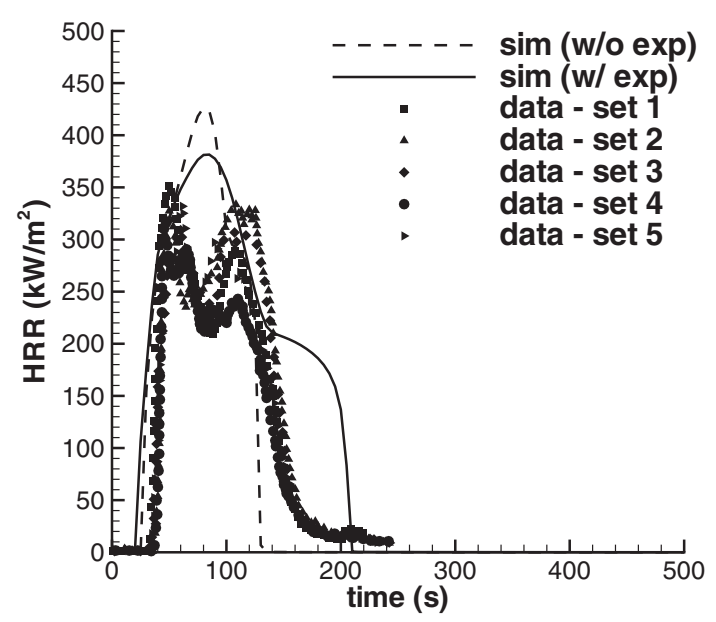

(c)

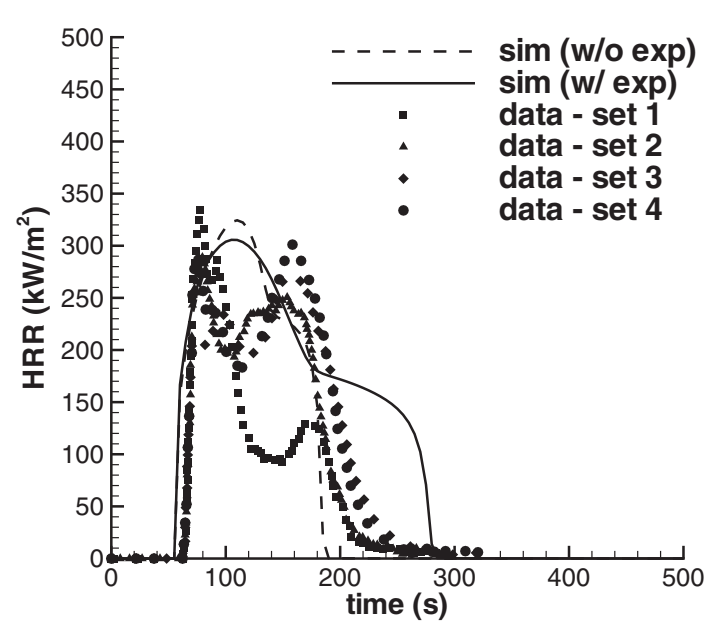

(b)

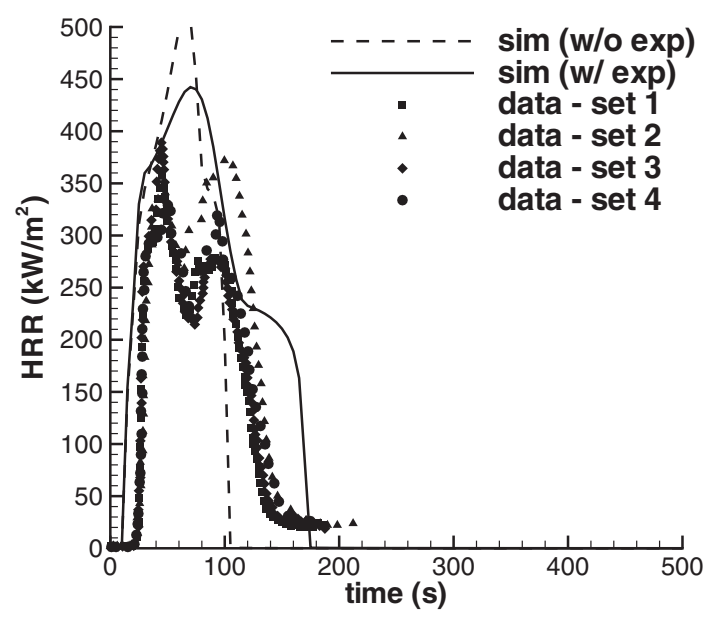

(d)

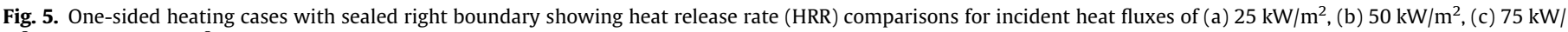
$\mathrm{m}^{2}$ and (d) $100 \mathrm{~kW} / \mathrm{m}^{2}$. 

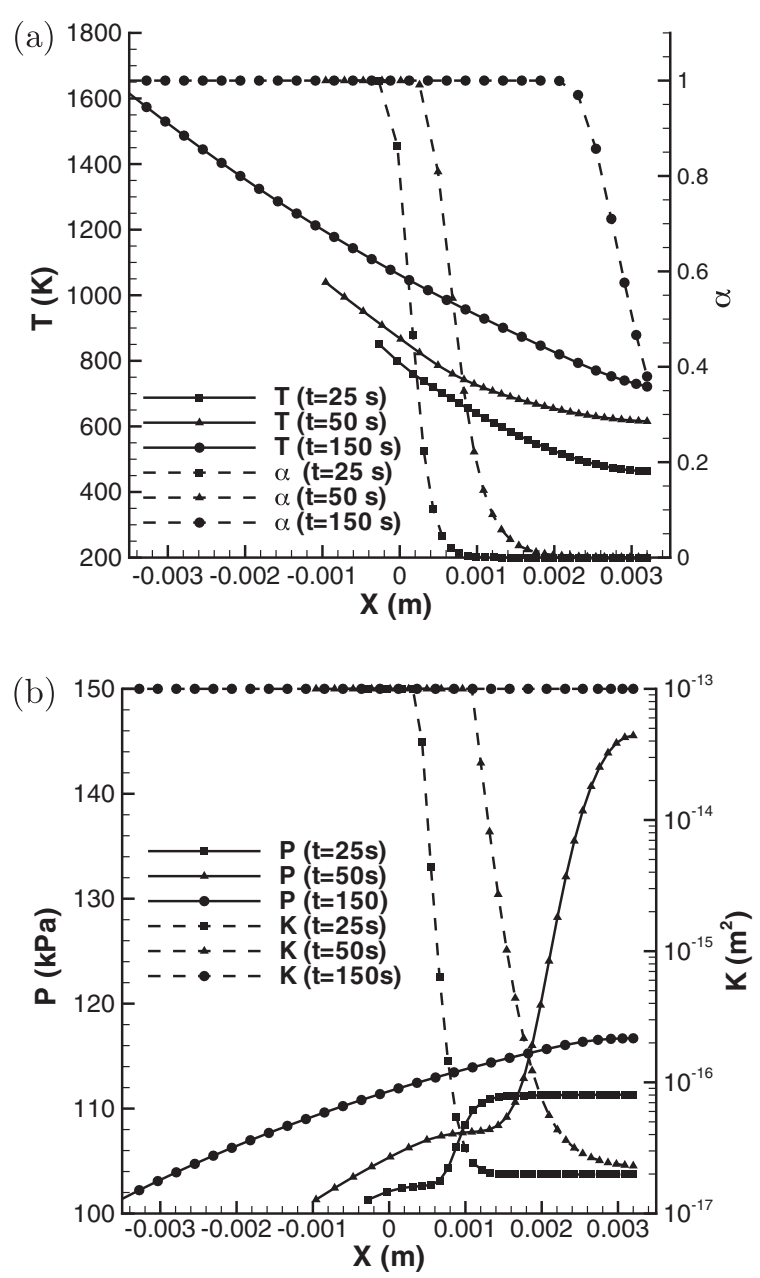

Fig. 6. Instantaneous snapshots of (a) $T \& \alpha$ and (b) $p_{g} \& K$ at $t=25,50$ and $75 \mathrm{~s}$. Symbols denote position of FE nodes.

the charred region due fiber clumping and/or collapse then the gas could potentially be limited enough to cause the more pronounced dip in HRR observed in the data. To account for these effects would require a much more sophisticated permeability model that depends on mechanical response. Nonetheless, the final product volume and mass fractions are well predicted as shown in Fig. 7 showing comparisons of (a) $V / V_{o}, V_{g} / V_{e} \& Y_{r c}$ and (b) $Y_{r c f}$ to data from QWC using the simulation results with the sealed right boundary. The overall agreement for $V / V_{o}, V_{g} / V_{e} \& Y_{r c}$ is quite good with errors less than $15 \%$ over the entire range of incident heat fluxes considered. The final mass predictions show in Fig. 7(b) appear to underpredict the data at lower heat fluxes, however, considering the repeatability uncertainty in the data it is difficult to draw definitive conclusions about deficiencies in the modeling.

\section{Conclusions}

A thermal model for a carbon-epoxy laminate is developed based on the data of Quintiere et al. [34]. The model includes pyrolysis decomposition, heat and mass transport, and volumetric swelling using a novel finite element algorithm. Model validation runs are conducted using TGA and one-sided heating experiments. Overall good agreement is observed between the model and data for the overall heat release rate and time-to-ignition. Neglecting the effects of the composite swelling resulted in significant under-predictions of flame burnout - highlighting the importance
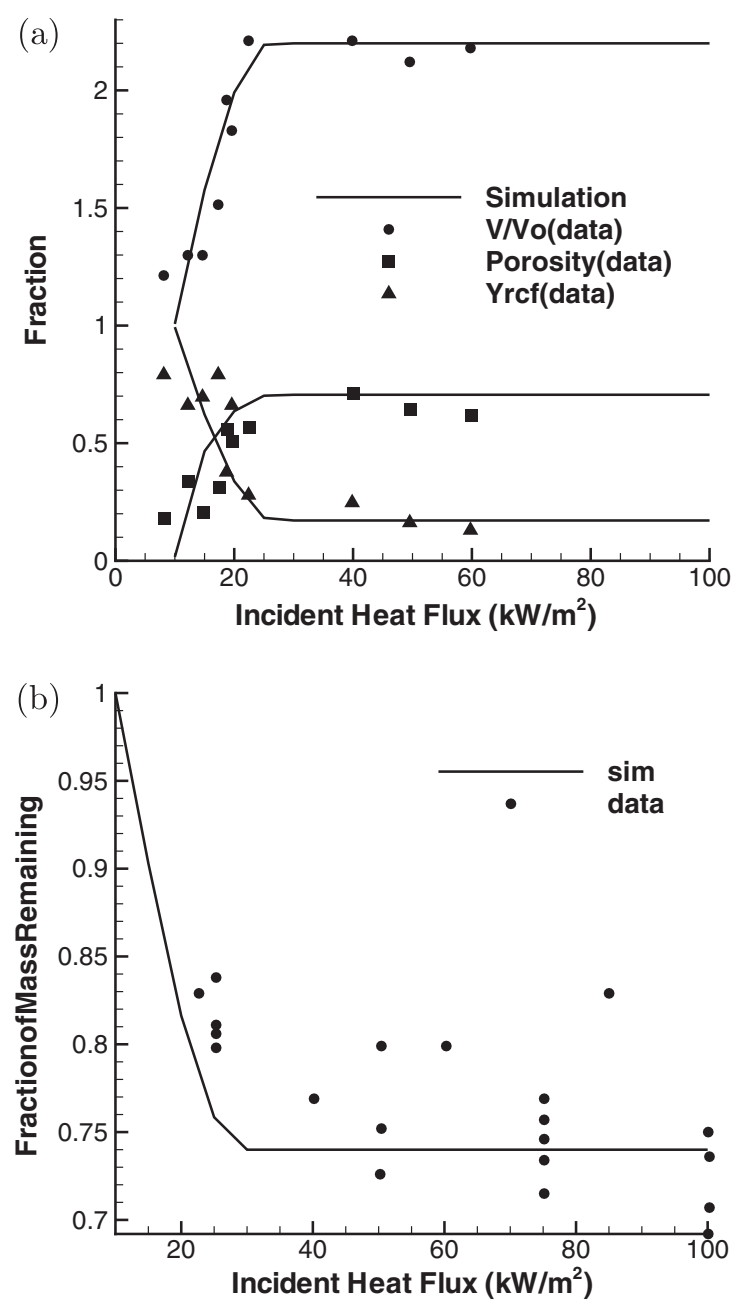

Fig. 7. Comparisons of predictions of (a) $V / V_{o}, V_{g} / V_{e} \& Y_{r c}$ and (b) $Y_{r c f}$ to data with increasing incident heat flux.

of accounting for laminate swelling for the current epoxy-carbon material. Remaining discrepancies in HRR predictions are attributed to three factors. The first is the uncertainty as to the specification of the unheated back boundary for the gas transport. Simulation results bound the HRR data using either an open or perfectly closed boundary indicting that the experimental conditions lie someplace in-between. This emphasizes the importance of being able to accurately characterize this boundary for future validation level experiments.

The second factor is the simplified estimate of HRR using the decoupled calculations that do not account for important gas-phase turbulent combustion processes. Future fully-coupled simulations will attempt to relax this assumption. The third factor is the permeability model which is monotonic through the decomposition front therefore does not account for potentially important matrix collapse processes that may change the time history of the HRR. However, the overall heat release predicted by the model seems to be in qualitative agreement with the data. Future efforts will try to quantify these differences by directly computing the overall heat released. Lastly, comparisons of the final volumetric expansion ratio, porosity and final char mass to the data are quite good, indicating that the overall thermal modeling approach is sound.

\section{Acknowledgements}

This research was supported by the Office of Naval Research under Grants N00014-06-1-0623 and N00014-08-C-0591, The 
National Science Foundation (NSF) under Grant CBET-1033328 and Sandia National Laboratories. Sandia National Laboratories is a multi-program laboratory operated by Sandia Corporation, a wholly owned subsidiary of Lockheed Martin Company, for the US Department of Energy's National Nuclear Security Administration under contract DE-AC04-94AL85000.

\section{References}

[1] C.A. Ulven, J. Mosbrucker, B. Miller, S. Pillay, S. Vaddi, U.K. Vaidya, Loss of balsa wood core structural integrity during fire exposure of sandwich composites, in: 38th SAMPE Fall Technical Conference: Global Advances in Materials and Process Engineering, International SAMPE Technical Conference, Soc. for the Advancement of Material and Process Engineering, Covina, CA 91724-3748, United States, 2006, p. 8.

[2] A. Mouritz, Z. Mathys, Post-fire mechanical properties of marine polymer composites, Composite Structures 47 (1999) 643-653.

[3] A. Mouritz, Z. Mathys, Post-fire mechanical properties of glass-reinforced polyester composites, Composites Science and Technology 61 (2001) 475-490.

[4] A. Mouritz, E. Gellert, P. Burchill, K. Challis, Review of advanced composite structures for naval ships and submarines, Composite Structures 53 (2001) 21 41.

[5] A. Mouritz, C. Gardiner, Compression properties of fire-damaged polymer sandwich composites, Composites 33 (2002) 609-620.

[6] A.G. Gibson, P.N.H. Wright, Y.S. Wu, A.P. Mouritz, Z. Mathys, C.P. Gardiner, The integrity of polymer composites during and after fire, Journal of Composite Materials 38 (2004) 1283-1307.

[7] G.A. Kardomateas, L. Liu, V. Birman, J.W. Holmes, G.J. Simitses, Thermal buckling of a heat-exposed, axially restrained composite column, Composites Part A (Applied Science and Manufacturing) 37 (2006) 972-980.

[8] J.B. Henderson, J.A. Wiebelt, M.R. Tant, A model for the thermal response of polymer composite materials with experimental verification, Journal of Composite Materials 19 (1985) 579-595.

[9] J.B. Henderson, T.E. Wiecek, A mathematical model to predict the thermal response of decomposing expanding polymer composites, Journal of Composite Materials 21 (1987) 373-393.

[10] J.J. Florio, J.B. Henderson, F.L. Test, R. Hariharan, A study of effects of the assummption of local-thermal equilibrium on the overall thermally-induced response of a decomposing glass-filled polymer composite, Journal of Heat and Mass Transfer 34 (1991) 135-146.

[11] R.M. Sullivan, N.J. Salamon, A finite element method for the thermochemical decomposition of polymeric materials-i. theory, International Journal of Engineering Science 30 (1992) 431-441.

[12] R.M. Sullivan, N.J. Salamon, A finite element method for the thermochemical decomposition of polymeric materials-ii. carbon phenolic composites, International Journal of Engineering Science 30 (1992) 939-951.

[13] R.M. Sullivan, A coupled solution method for predicting the thermostructural response of decomposing, expanding polymeric composites, Journal of Composite Materials 27 (1993) 408-434.

[14] G.A. Pering, P.V. Farrell, G.S. Springer, Degradation of tensile and shear properties of composites exposed to fire or high temperature, Journal of Composite Materials 14 (1980) 54-68.

[15] H.L.N. McManus, G.S. Springer, High temperature thermomechanical behavior of carbon-phenolic and carbon-carbon composites, i. analysis, Journal of Composite Materials 26 (1992) 206-229.

[16] H.L.N. McManus, G.S. Springer, High temperature thermomechanical behavior of carbon-phenolic and carbon-carbon composites, ii. results, Journal of Composite Materials 26 (1992) 230-255.
[17] Y.I. Dimitrienko, Thermomechanical behavior of composite materials, structures under high temperatures: 1. materials, Composites Part A 28 (1997) 453-461.

[18] Y.I. Dimitrienko, Thermomechanical behavior of composite materials and structures under high temperatures: 2. structures, Composites Part A 28 (1997) 463-471.

[19] Y.I. Dimitrienko, Internal heat-mass transfer and stresses in thin-walled structures of ablating materials, International Journal of Heat Mass Transfer 40 (1997) 1701-1711.

[20] Y.I. Dimitrienko, Thermomechanical behavior of composites under local intense heating by irradiation, Composites: Part A 31 (2000) 591-598.

[21] A.G. Gibson, Y.S. Wu, H.W. Chandler, J.A.D. Wilcox, P. Bettess, Model for the thermal performance of thick composite laminates in hydrocarbon fires, Revue de l'Institute Francais du Petrole 50 (1995) 69-74.

[22] A. Mouritz, S. Feih, E. Kandare, Z. Mathys, A.G. Gibson, P. DesJardin, S.W. Case, L.B.Y., Review of fire structural modelling of polymer composites, Composites 40 (2009) 1800-1814.

[23] L. Couchman, A.P. Mouritz, Modeling of Naval Composite Structures in Fire, CRC for Advanced Composite Structures, Templestowe, Victoria, Australia, 2006.

[24] A.P. Mouritz, A.G. Gibson, Fire Properties of Polymer Composite Materials, Solid mechanics and its applications, Springer, Dordrecht, The Netherlands, 2006.

[25] C. DiBlasi, The state of the art of transport models for charring solid degradiation, Polymer International 49 (2000) 1133-1146.

[26] C. Di Blasi, Modeling chemical and physical processes of wood and biomass pyrolysis, Progress in Energy and Combustion Science 34 (2008) 47-90.

[27] C. Lautenberger, C. Fernandez-Pello, Generalized pyrolysis model for combustible solids, Fire Safety Journal 44 (2009) 819-839.

[28] C. Lautenberger, Gpyro A Generalized Pyrolysis Model for Combustible Solids Technical Reference Guide, 0.659 edition, 2009.

[29] K. McGrattan, S. Hostikka, J. Floyd, H. Baum, R. Rehm, Fire Dynamics Simulator (Verion 5) Technical Reference Guide, National Institute of Standards and Technology, 2007.

[30] S.I. Stoliarov, R.E. Lyon, Thermo-Kinetic Model of Burning, Technical Report DOT/FAA/AR-TN08/17, US Department of Transportation, Federal Aviation Administration, 2008.

[31] S.I. Stoliarov, R.E. Lyon, Thermo-kinetic model of burning for pyrolyzing materials, in: Fire Safety Science 9, pp. 1141-1152.

[32] C. Luo, P.E. DesJardin, Thermo-mechanical damage modeling for a glass-fiber phenolic-resin composite material, Composites Science and Technology 67 (2007) 1475-1488.

[33] J.E.J. Staggs, A simple model of polymer pyrolysis including transport of volatiles, Fire Safety Journal 34 (2000) 69-80.

[34] J.G. Quintiere, R.N. Walters, S. Crowley, Flammability Properties of Aircraft Carbon-Fiber Structural Composite, Technical Report DOT/FAA/AR-07/57, US Department of Transportation, Federal Aviation Administration, 2007.

[35] F. Kuwahara, Y. Kameyama, S. Yamashita, A. Nakayama, Numerical modeling of turbulent flow in porous media using a spatially periodic array, Journal of Porous Media 1 (1998) 47-55.

[36] R. Kee, F. Rupley, J. Miller, The CHEMKIN Thermodynamic Data Base, Technical Report, Sandia National Laboratory, 1987.

[37] K. Erickson, Thermal decomposition mechanisms common to polyurethane, epoxy, poly(diallyl phthalate), polycarbonate and poly(phenylene sulfide), Journal of Thermal Analysis and Calorimetry 89 (2007) 427-440.

[38] C.L. Beyler, M.M. Hirschler, Thermal decomposition of polymers, in: P. DiNenno (Ed.), The SFPE Handbook of Fire Protection Engineering, National Fire Protection Association, 2002. pp. 1-110 - 1-131.

[39] A. Tewarson, Generation of heat and chemical compounds in fires, in: P. DiNenno (Ed.), The SFPE Handbook of Fire Protection Engineering, National Fire Protection Association, 2002. pp. 3-82 - 3-161.

[40] J.N. Reddy, Finite Element Method, McGraw Hill, New York, NY, 1993. 